\title{
Depression and Parental Bonding: Cause, Consequence, or Genetic Covariance?
}

\author{
Michael C. Neale, Ellen Walters, Andrew C. Heath, Ronald C. Kessler, \\ Daniel Pérusse, Lindon J. Eaves, and Kenneth S. Kendler
}
Departments of Human Genetics (M.C.N., D.P., L.J.E., K.S.K.) and Psychiatry (E.W., L.J.E., K.S.K.), Medical College of Virginia, Richmond; Department of Psychiatry, Washington University School of Medicine, St. Louis, Missouri (A.C.H.); Institute for Social Research, University of Michigan, Ann Arbor (R.C.K.)

\begin{abstract}
It is shown how information on the direction of causation between variables may be obtained from a cross-sectional study of pairs of relatives. This method is applied to the study of the relationship between ratings of parents' rearing style and depression in their offspring. Adult female twins ascertained from a population-based registry in Virginia completed the Center for Epidemiological Studies - Depression Scale (CESD) and a 7-item short form of the Parental Bonding Instrument (PBI) about each of their parents. Two dimensions of parental behavior, overprotectiveness and coldness, were analyzed jointly with depression data in both genetic factor and directional genetic models. Models that specify ratings of parents as a cause of depression in the offspring fit the data significantly better than models that specify depression as a cause of ratings of parents. A still better fit is obtained with models that specify common genetic variance to depression and ratings, though causal models with error variance perform almost as well. In general, ratings of fathers show more genetic and less shared environmental variance than ratings of mothers, which might arise from more consistent treatment of offspring by mothers than by fathers. No effect of children eliciting parental rearing style was detected with these data. The relative merits of instrumental variable, longitudinal, and family approaches to testing causal models are discussed. (c) 1994 Wiley-Liss, Inc.
\end{abstract}

Received for publication July 31, 1991; revision accepted March 15, 1992.

Address reprint requests to Michael C. Neale, Department of Human Genetics, Medical College of Virginia, Box 3, Richmond, VA 23298.

(C) 1994 Wiley-Liss, Inc. 
Key words: depression, CESD, psychiatry, genes, environment, twins, parents, overprotectiveness, bonding, coldness, Parker, ratings, causation, longitudinal, instrumental variable, design, methodology, structural equation modeling, $\mathbf{M x}$

\section{INTRODUCTION}

Studies in several countries have shown significant and substantial relationship between measures of depression and ratings of parental rearing style. The association has been found within both clinical samples [Parker, 1979a; Plantes et al., 1988; Birtchnell, 1988] and non-clinical groups such as college students [Parker, 1979b] and volunteer twins [Parker, 1986], although it was not replicated in a general sample [Mackinnon et al., 1989]. Two principal dimensions of parental style have been the focus of most research to date: a caring or warmth factor and an overprotectiveness dimension. Both dimensions may be assessed with the self-report Parental Bonding Instrument (PBI) [Parker, 1979b]. Neurotic (or unipolar) depressives perceive both parents as being less caring than do either controls or manic depressives [Jacobson et al., 1975; Parker, 1979a,b; Parker et al., 1987; Gotlib et al., 1988; Plantes et al., 1988; Alnaes and Torgerson, 1990]. In addition, mothers - but not fathers- of neurotic depressives are perceived as being more overprotective than mothers of controls or manic patients [Parker, 1979a] or endogenous depressives [Parker et al., 1987]. Among non-clinical groups, correlations between Costello-Comrey trait depression scores [Costello and Comrey, 1967] and parental style variables range from -0.15 to -0.45 , and similar findings have been found with other measures of parental rearing style [CRPBI: Raskin et al., 1971; EMBU: Perris et al., 1985; HEI: Holmes and Robins, 1987; FES: Plomin et al., 1988]. Given the strength and significance of these associations, it becomes important to investigate whether 1) parental style is a genuine environmental risk factor for depression, or 2) ratings of parents are biased by the reporting styles of depressed and non-depressed subjects [Beck et al., 1979], or 3) they correlate due to some unmeasured factor such as parental depression or pleiotropic genes [Holmes and Robins, 1987].

Available data suggest that parental style, not rating style, is the source of the association with depression. First, ratings of parents were obtained from patients during an episode of depression and subsequently in remission. No significant differences between occasions were found in two studies [Parker, 1981; Gotlib et al., 1988], but Lewinsohn and Rosenbaum [1987] found that the remitted depressed did not differ from controls. With this exception, however, ratings of parental behavior seem remarkably stable in both non-clinical [Wilhelm and Parker, 1990] and clinical samples. Second, children's ratings of their parents significantly correlate approximately 0.5 with parents' self ratings of their treatment of the children [Parker, 1981]. Moreover, siblings and twins show substantial agreement in their ratings of their parents [Robins et al., 1985; Parker, 1986]. Thus reports of rearing style seem to possess the psychometrically desirable qualities of reliability and validity. Although these findings are consistent with a causal effect of parents' rearing style on children's liability to depression, several alternative hypotheses are not excluded. It is still possible that rating style differs between depressives in remission and normal controls. Another possibility is that the premorbid depressive child elicits low caring and overprotective rearing styles from his or her parents. Chodoff [1972] has suggested that dependency traits are common precursors to depression. However, correlations 
between PBI scales and depression measures are not altered by partialing out measures of dependency or shyness [Parker, 1981]. Although encouraging, these data do not rule out elicitation of rearing styles associated with depression; there is little substantive knowledge about the premorbid depressive personality [Becker, 1974], and other, unmeasured aspects of the child may influence parental behavior.

What other data might be collected to examine the association between depression and parenting? In many areas of science, experimentation is the method of choice for testing causal models. Subjects are randomly assigned to separate groups and the hypothetically causal variable of interest is directly manipulated in a different way in the various groups. In the case of rearing style and depression, there are numerous problems with this approach. First, it would be unethical to request parents to change their behavior in directions that may increase psychopathology in their children. Second, parents may be unwilling or unable to alter their behavior at all. Third, we do not know whether depression is caused by parental coldness for a short, sensitive period, or gradually during years of development of the child. Especially in the latter case, the artificial modification of parents' behavior would be unlikely to map closely to behavior of naturally warm parents. Experimentation is inappropriate in many areas of social science, and the study of rearing behavior is no exception.

When experimentation is unethical or impractical, naturalistic observation often provides a suitable alternative strategy to test causal hypotheses. At simplest level, we could reject the hypothesis that cold parental rearing style causes depression if the two variables were independent. The studies described above do not reject the hypothesis at this level, and further tests are warranted. One such test would be to measure subjects on more than one occasion in a longitudinal design. This method has considerable merit, since by definition cause precedes effect and causation from measures on the second occasion to measures on the first occasion may be excluded a priori. Early approaches [e.g., Campbell, 1963] compared across-occasion acrossvariable correlations. For example, the correlation of parental rearing at age 12 years with depression at age 16 years would be compared with the correlation of depression at age 12 years with parental rearing at age 16 years. More formal hypothesis testing in this design (often called cross-lagged panel analysis) was developed using path analysis [e.g., Heise, 1975; Kessler and Greenberg, 1981; Mayer, 1984; Werts et al., 1971, 1977, 1985] (see Fig. 1). If the correlation of rearing at time 1 with depression at time 2 did not significantly exceed the product of the time 1 rearingdepression correlation and the across-occasion depression correlation, then the hypothesis that rearing style causes depression during this period of development would be rejected. But in order for longitudinal studies to detect causation, the causal process must be occurring between the occasions of measurement [Biddle et al., 1985]. This limitation makes the longitudinal study a rather unattractive method for assessing the relationship between rearing and depression, because depression often does not have onset until adulthood, when children are no longer living with their parents. Particularly for early childhood rearing style, data on depression would effectively be missing on the earlier occasion of measurement since onset rarely occurs before puberty [Rutter, 1986].

A second approach to testing causal models with observational data involves the measurement of instrumental variables [e.g., Theil, 1971]. Suppose that wealthy parents were more able to be caring about their children than less fortunate parents. 


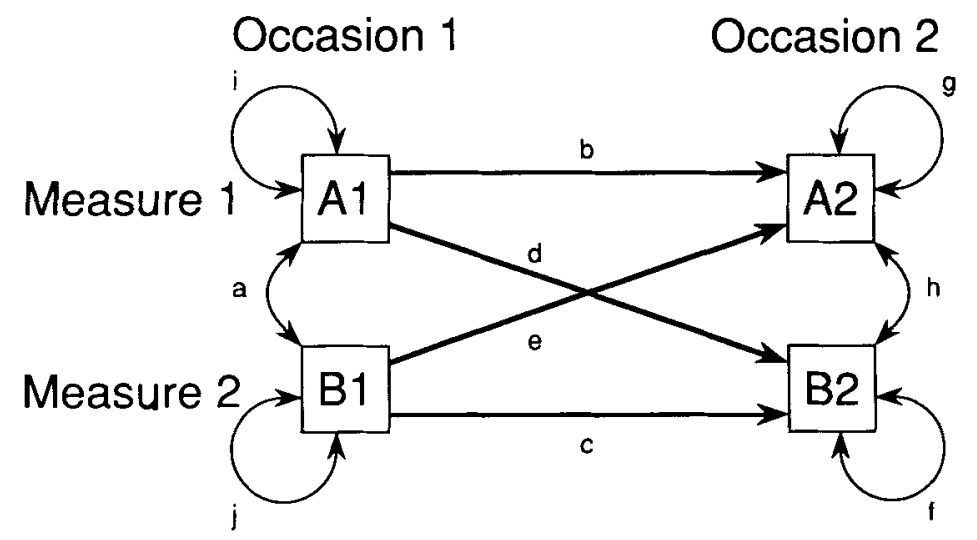

Fig. 1. Cross-lagged panel path model. Data on 2 variables (A and B) are collected on 2 occasions from a sample of unrelated individuals. Residual variances ( $, \mathrm{j}, \mathrm{f}$, and $\mathrm{g}$ ) and covariances $(\mathrm{a}$ and $\mathrm{h}$ ) are shown with double-headed arrows. Tests for the significance of parameters $d$ and e assess causation between these variables between occasions 1 and 2 .

If low parental care is a cause of depression, then low income parents should have more depressed children than high income parents. However, if offspring depression (or correlated factors in the offspring) were the cause of parental rearing style, no such association with income would be observed (see Fig. 2). Obviously this approach depends on some critical assumptions, e.g., that rearing style and children's
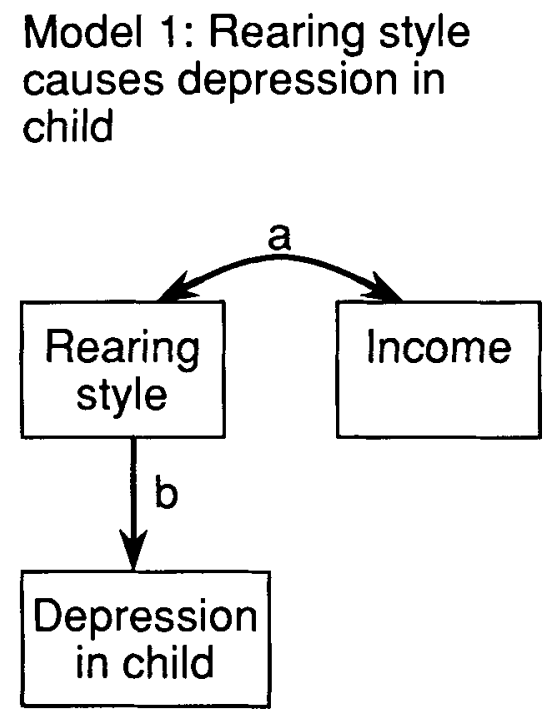

Parental income correlates with child's depression
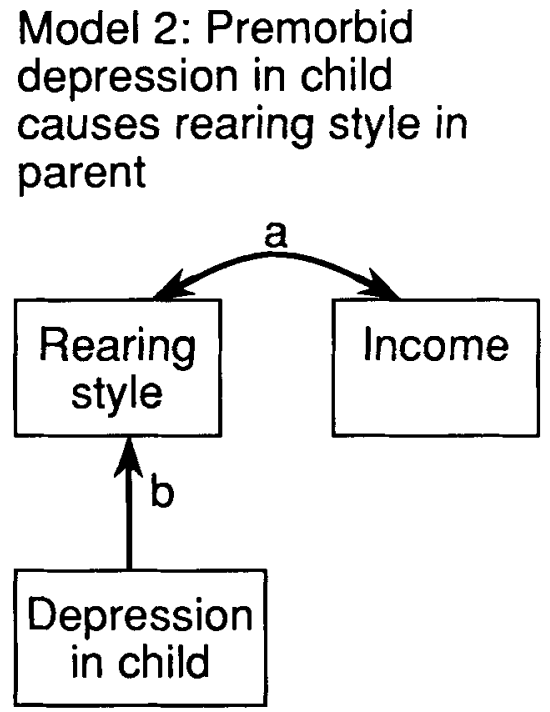

Parental income does not correlate with child's depression

Fig. 2. Use of instrumental variables to discriminate between directional models. 
depression are not a cause of income. The unambiguous identification of instrumental variables is not a simple matter in the social sciences where genetic or environmental factors or both may covary between variables.

A third approach to assess causation is to use data collected from relatives on a single occasion of measurement [Heath et al., 1989; Duffy and Martin, 1992]. Despite the absence of repeated measures, the strategy is formally similar to the longitudinal or cross-lagged panel approach described above (see Fig. 3). With two variables measured in paris of relatives, there are four observed variables in the system. In contrast to the longitudinal approach, we cannot exclude paths from the model because of temporal precedence. Instead, we assume that the members of a pair of relatives are not having any mutual effect on each other. This assumption is equivalent to the one made in cross-lagged panel analysis that there are no mediating variables (i.e., residuals are uncorrelated). In addition, we make the natural assumption that the relationship between the variables is the same for relative 1 as it is for relative 2 (see Fig. 3). Identification of the model also requires that the correlation between relatives is different for the two variables, (i.e., $a \neq d$ in Fig. 3). In striking contrast to the longitudinal design, if variable $X$ were a cause of variable $Y$ prior to the occasion of measurement, this process still would be revealed in the pattern of across-variable across-relative correlations. In this respect, the information offered by a study of pairs of relatives is extremely valuable for the study of rearing style and depression. Such a study was undertaken with 39 pairs of twins from the Australian National Health and Medical Research Council Registry [Parker, 1986]. In addition to replicating the association between rearing style and depression, the study revealed approximately 0.7 correlation between twins for their ratings of their parents, regardless of zygosity. Across-twin across-variable correlations were not reported, but it would not be easy to discriminate between models for the relationship between the variables with data from such a small sample.

In this report we present and analyze data from a large study of female twins in Virginia, assessed with a short form of the PBI and a different measure of depression.

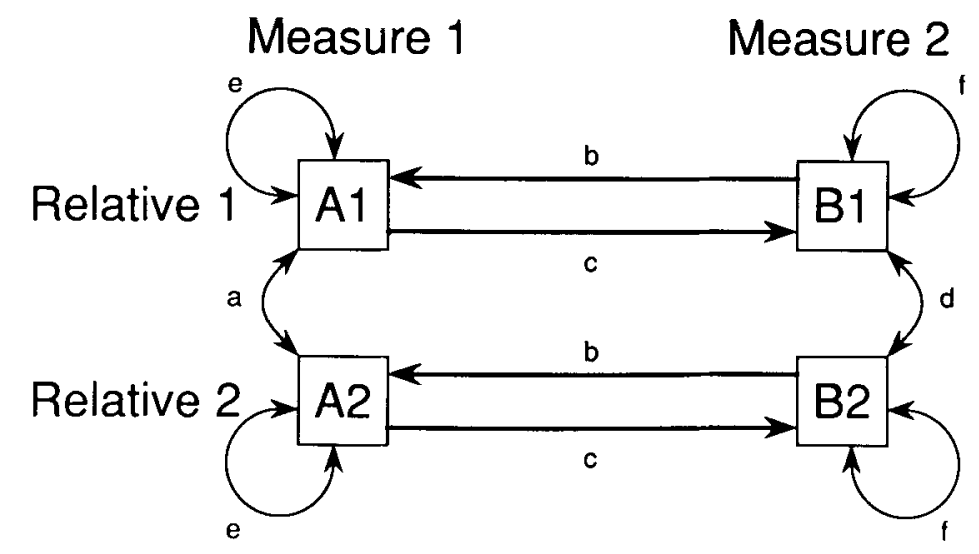

Fig. 3. Directional path model for a pair of relatives measured in 2 variables ( $A$ and $B$ ). Relatives correlate within variables (paths a and d) due to genetic or shared environmental factors. Within-person variables may correlate due to causal paths b or $\mathrm{c}$ or both; all variables have residual variance represented by paths $\mathrm{e}$ and f. Across-variable, across-person correlations (e.g., Al, B2) are predicted by the model. 
Our aims are 1) to assess the role of genetic and environmental factors in ratings of rearing style; 2) to test models for direction of causation between depression and rearing style measures; and 3) to test whether children elicit parental behavior.

\section{METHODS}

\section{Measures}

Assessment of parental rearing style was not the primary aim of the data collection undertaken in our study of anxiety and depression. Due to severe space limitations, only 7 items on rearing style could be assessed within the questionnaire schedule. Items $(3,4,5,7,16,19$, and 23) were selected from the original PBI with the aim of constructing a short questionnaire with a balance between positivity and negatively weighted items for each factor. In practice, factor analysis showed that item 7 loaded more strongly on the coldness factor, so scores were computed by summing item responses (or 5-item response for negatively weighted items). Coldness scale scores were imputed (see Appendix) for individuals who answered over $50 \%$ of items. No imputation was used for the 2 overprotection items. The 20-item Center for Epidemiological Studies - Depression (CESD) scale [Radloff, 1977] was included in the same questionnaire together with several other scales assessing personality, anxiety, and depression. CESD item responses were summed; data were imputed if subjects failed to answer from 1 to 9 items. The resulting scale exhibits marked skewness (observed in other population samples [Comstock and Helsing, 1976]) which is not entirely removed by $\log$ transformation. Therefore, we chose to work with an assumed underlying normal distribution of liability to depression, with 9 thresholds giving rise to 10 classes of depressive phenotype. The assumption of a normally distributed liability follows from the central limit theorem [Mardia et al., 1979], which postulates additive action of an infinite number of independent factors, each of small effect. In practice, quite a small number of factors will give rise to a distribution that is close to normal [Kendler and Kidd, 1986].

\section{Subjects}

Data for this report come from a current study of genetic and environmental risk factors for common psychiatric disorder in Caucasian female same-sex twin pairs from the population-based Virginia Twin Registry. The mean age of the sample was $30.1 \pm 7.6$; further details of the sample are given in Kendler et al. [1991a,b]. Zygosity determination was made using questionnaires and photographs and cases still in doubt following this procedure were assessed using DNA polymorphisms. Thus the misclassification rate is likely to be extremely low.

\section{RESULTS}

\section{Endorsement Frequencies and Summary Statistics for Model Fitting}

In view of the large sample size of this study $(N=1,680)$, endorsement frequencies for the CESD items and PBI items are shown in Tables I and II. Note that nearly half the sample felt depressed for at least 1 day during the past week, but that less than a quarter of the sample felt that people disliked them on at least 1 day. There is also considerable variability in rearing styles, although relatively few parents seem to make their children feel not wanted. 
TABLE I. Endorsement Frequencies for CESD Items Completed by Adult Female Twins in Virginia

\begin{tabular}{lrrrrrr}
\hline & \multicolumn{7}{c}{ Days } & & & \\
\cline { 2 - 4 } CESD item & \multicolumn{1}{c}{$r$} & $1-2$ & $3-4$ & $5-7$ & Total & Missing \\
\hline Bothered by things & 1,099 & 384 & 135 & 50 & 1,668 & 12 \\
Poor appetite & 1,157 & 326 & 136 & 53 & 1,672 & 8 \\
Felt blue & 1,142 & 306 & 146 & 72 & 1,666 & 14 \\
Felt as good as others & 180 & 210 & 349 & 904 & 1,643 & 37 \\
Trouble concentrating & 849 & 507 & 217 & 73 & 1,646 & 34 \\
Depressed & 860 & 510 & 189 & 104 & 1,663 & 17 \\
Everything an effort & 1,037 & 414 & 142 & 75 & 1,668 & 12 \\
Hopeful about future & 226 & 322 & 442 & 681 & 1,671 & 9 \\
Life a failure & 1,417 & 177 & 44 & 25 & 1,663 & 17 \\
Felt fearful & 1,387 & 203 & 52 & 27 & 1,669 & 11 \\
Restless sleep & 792 & 496 & 256 & 123 & 1,667 & 13 \\
Felt happy & 100 & 237 & 483 & 839 & 1,659 & 21 \\
Talked less than usual & 870 & 523 & 212 & 56 & 1,661 & 19 \\
Felt lonely & 1,064 & 375 & 134 & 98 & 1,671 & 9 \\
People unfriendly & 1,310 & 274 & 60 & 26 & 1,670 & 10 \\
Enjoyed life & 85 & 228 & 448 & 907 & 1,668 & 12 \\
Crying spells & 1,271 & 246 & 103 & 43 & 1,663 & 17 \\
Felt sad & 944 & 514 & 147 & 62 & 1,667 & 13 \\
People dislike me & 1,314 & 261 & 58 & 33 & 1,666 & 14 \\
Couldn't get going & 892 & 549 & 164 & 66 & 1,671 & 9 \\
\hline
\end{tabular}

Two types of summary of the data were used for model fitting: 1) contingency tables of twins' responses (univariate analyses) and 2) matrices of polychoric correlations and associated weight matrices (bivariate analyses). Both types of summary were computed for the 5 measures: CESD, maternal coldness (MOCOLD), maternal overprotectiveness (MOOVER), paternal coldness (FACOLD), and paternal overprotectiveness (FAOVER). CESD responses were divided into 10 categories; the uppermost 3 categories were combined to generate tables without a large number of cells with zero observed frequency. The coldness and overprotection scales had, respectively, 7 and 6 categories of response. The 10 contingency tables of monozy-

TABLE II. Endorsement Frequencies for Parker Items

\begin{tabular}{lcrrrrrr}
\hline Item & Parent & A lot & Some & Little & None & Total & Missing \\
\hline \multirow{2}{*}{ Let me do things l liked } & Mom & 717 & 620 & 275 & 48 & 1,660 & 20 \\
& Dad & 665 & 604 & 287 & 65 & 1,621 & 59 \\
Emotionally cold to me & Mom & 105 & 156 & 259 & 1,139 & 1,659 & 21 \\
Understood my problems & Dad & 166 & 196 & 320 & 944 & 1,626 & 54 \\
& Mom & 569 & 544 & 369 & 180 & 1,662 & 18 \\
Liked me to make my own decisions & Dad & 352 & 502 & 478 & 290 & 1,622 & 58 \\
& Mom & 453 & 667 & 385 & 155 & 1,660 & 20 \\
Made me feel not wanted & Dad & 481 & 581 & 387 & 172 & 1,621 & 59 \\
& Mom & 71 & 90 & 180 & 1,321 & 1,662 & 18 \\
Made me dependent on her & Dad & 117 & 65 & 179 & 1,259 & 1,620 & 60 \\
& Mom & 139 & 201 & 328 & 992 & 1,660 & 20 \\
Overprotective of me & Dad & 88 & 156 & 296 & 1,080 & 1,620 & 60 \\
& Mom & 333 & 355 & 446 & 526 & 1,660 & 20 \\
& Dad & 263 & 319 & 455 & 583 & 1,620 & 60 \\
\hline
\end{tabular}


gotic (MZ) and dizygotic (DZ) twins' responses on the 5 scales are available on request. The correlation matrices were computed pairwise for the twins, yielding the two $(10 \times 10)$ correlation matrices shown in Table III. The within-person correlations between CESD and the ratings of parental rearing style consistently indicate greater reported emotional coldness and overprotectiveness among subjects with greater selfreported depression. MZ twin correlations are almost invariably greater in absolute magnitude than the corresponding DZ twin correlations, suggesting a role for genetic factors in the variance of both depression and ratings. Ratings of mother and father made by the same individuals correlate substantially (approximately 0.8 for overprotectiveness and 0.6 for coldness). The asymptotic variances and covariances of the correlations were calculated using PRELIS 1.20 [Jöreskog and Sörbom, 1986]; since these matrices are of order $45 \times 45$, they are not reproduced here but are available on request. Regressions on age accounted for less than $1 \%$ of the variance of all measures, and age was not included in subsequent modeling [Neale and Martin, 1989].

Path models are diagrams of causal and correlational relationships between latent and observed variables [McArdle and Boker, 1990]. A set of tracing rules may be used to derive expectations for observed variances and covariances between variables [e.g., see Heise, 1975; Li, 1975; Bollen, 1989; Neale and Cardon, 1992]. A second method, used here, is to formulate the model in terms of a quadratic matrix product, which generates the expected covariance matrix between all the observed variables for a group. We use two different strategies for model fitting. For the univariate analyses, models were fitted by maximum likelihood directly to the two-way contingency tables of twin 1 response against twin 2 response [Neale et al., 1986; Neale, 1988]. Thus goodness-of-fit statistics reflect 3 components: 1) the adequacy of the model for covariance structure, 2) the adequacy of the assumption that thresholds do not differ according to zygosity or order of twins; and 3) the assumption that liability is bivariate normal. Mx fits threshold models to two-way tables only, so we used a different strategy to fit the bivariate models. Prediction of the observed correlation matrices was optimized for each model and submodel by minimizing a weighted least squares loss function that is a measure of the discrepancy between the observed and expected statistics [AWLS; Browne, 1984]. All models were fitted using Mx, a package for multivariate analysis of genetically informative data [Neale, 1991].

\section{Univariate Genetic Models}

To test whether the univariate data were consistent with the assumption of an underlying bivariate normal distribution of liability, we fitted a "full" model in which twins' thresholds were allowed to differ both between $\mathrm{MZ}$ and DZ twins and between twin 1 and twin 2 within these groups. The correlation in liability was allowed to take any value between -1 and 1 in each group. Table IV shows the results of fitting this model, in the column marked "Full", the $\chi^{2}$ statistic is clearly non-significant for depression and maternal overprotection, is borderline for maternal and paternal coldness, and is significant for paternal overprotection. Examination of the groups separately reveals that the DZ group is well approximated by the bivariate normal, whereas the MZ group has an excess of perfectly concordant pairs, suggesting a leptokurtic distribution for these data.

A simple model consisting of additive genetic (A), common environment (C), and random environment (E) components of variance [Heath et al., 1989] was 


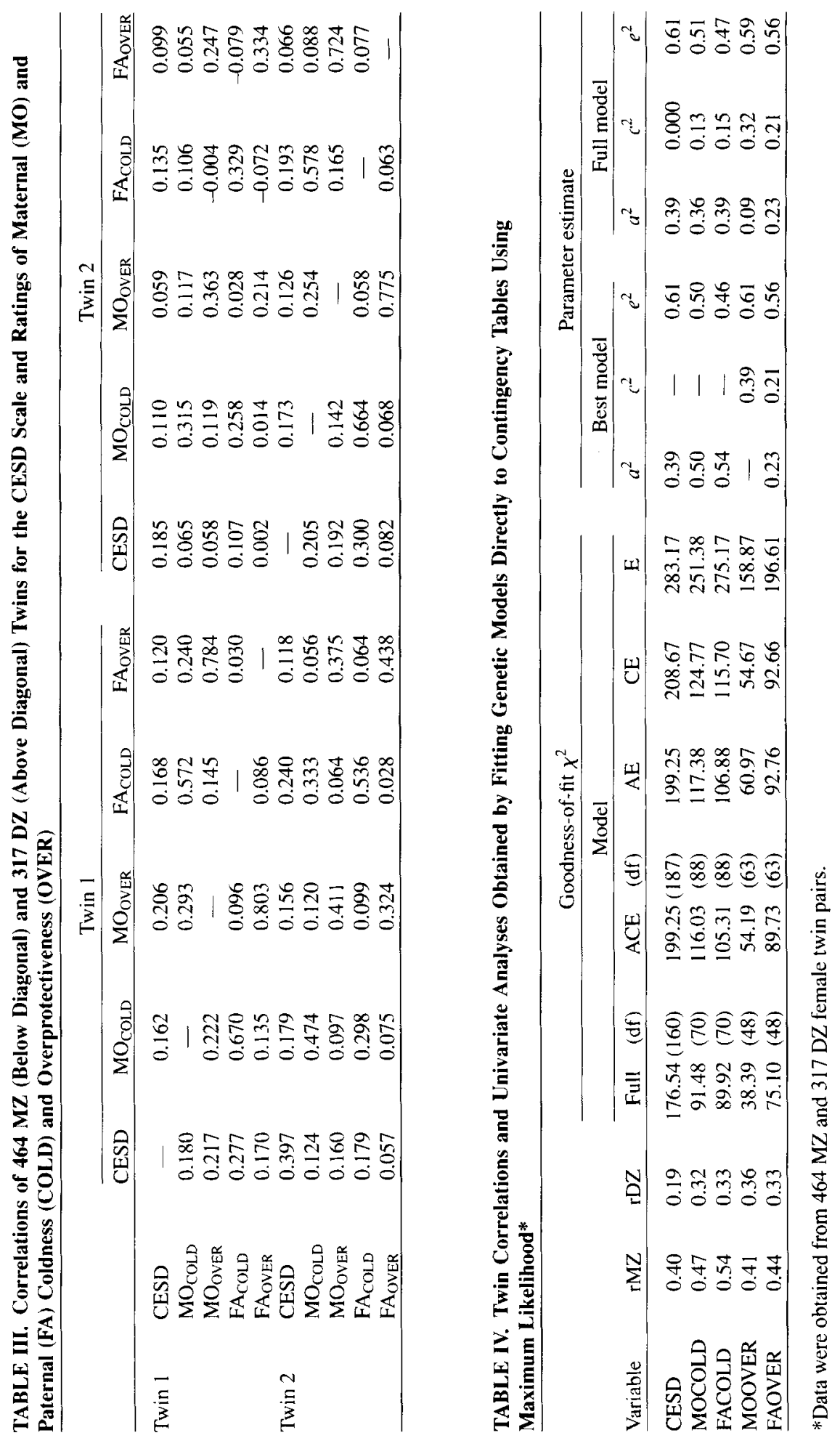


fitted to the same contingency tables; thresholds were equated across zygosities and across twin 1 and twin 2 . Total phenotypic variances (i.e., $a^{2}+c^{2}+e^{2}$ ) were constrained to equal unity, since (within groups) total variance is confounded with the threshold parameters. Hypothesis testing was carried out using what is called the likelihood ratio or $\chi^{2}$ difference test. In general, suppose we have two models, $M_{1}$ and $M_{2}$, with respective goodness-of-fit $\chi^{2}$ statistics $G_{1}$ and $G_{2}$, and respective degrees of freedom $d_{1}$ and $d_{2}$. Further, let $M_{2}$ be a submodel of $M_{1}$, i.e., it is the same model but has one or more parameters fixed to a particular value ex hypothesi or has one or more parameters set equal to a function of other parameters. For the log-likelihood and asymptotic weighted least squares fit statistics used here, the difference between the respective goodness-of-fit statistics, $G_{2}-G_{1}$ is itself asymptotically distributed as $\chi^{2}$ with $d_{2}-d_{1}$ degrees of freedom. We apply this theory to test the difference between the full model and the ACE model (i.e., column 4 vs. column 3 in Table IV). The difference is non-significant for all 5 variables, so the hypotheses that 1 ) thresholds do not differ as a function of zygosity or twin and 2) the pattern of correlations is not significantly deviant from those predicted by the ACE model are not rejected. Submodels of the ACE model were fitted to assess the statistical significance of the parameters $A$ and $C$. The results (Table IV) indicate moderate genetic influence in 4 of the 5 scales, with no evidence for genetic factors in ratings of maternal overprotectiveness. Common environmental factors are estimated to be non-zero for the ratings of parents, but are non-significant by $\chi^{2}$ difference test for all scales except maternal overprotectiveness. Specific environmental factors are associated with $45-61 \%$ of phenotypic variability, with depression having the largest proportion of this source of variance.

\section{Bivariate Directional Models}

Bivariate directional models incorporating genetic and environmental factors $\mathrm{A}$, $\mathrm{C}$, and E (see Fig. 4) were fitted to the CESD data with the 4 parental rearing style variables. In each case, 9 models were fitted: a depression-causes-ratings model; a ratings-causes-depression model; these two models but incorporating error of measurement for the causal variable; a bidirectional causal model; an independent pathways model, allowing additive genetic, common, and specific factors for depression to affect ratings of parents; and three submodels of this model, each having only one source of covariation specified. The independent pathways model involves 3 parameters to account for the 3 covariances between variables (within persons, across MZ twins, and across DZ twins). The bidirectional model and unidirectional models with error use 2 parameters and the remaining models use only 1 . In general, the model with most parameters is likely to fit the data better (i.e., to have the lowest $\chi^{2}$ ), but we seek parsimony as well as absolute goodness-of-fit in our explanation of the data [Neale et al., 1989]. One approach to the comparison of models that reflects both economical use of parameters and goodness-of-fit is Akaike's information Criterion (AIC) [Akaike, 1987], which is calculated here as $\chi^{2}-2 d f$. The lowest (i.e., largest negative) values of this index provide the most parsimonious explanation of the data.

Results of model fitting are shown in Tables V and VI. In absolute terms, the only models rejected by the data are the unidirectional causal and common $\mathrm{C}$ models for paternal coldness, and the common E models. By Akaike's [1987] information criterion, the most parsimonious model is invariably the common genetic factor model, 


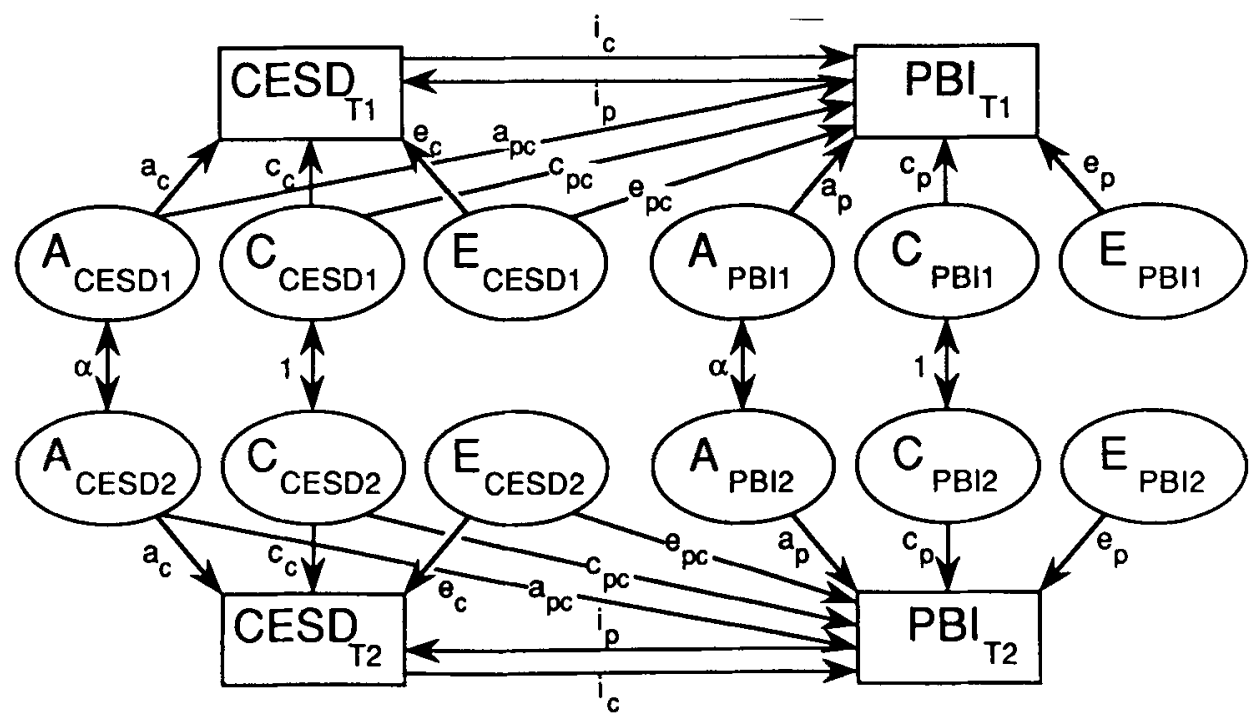

Fig. 4. Underidentified model for bivariate twin resemblance. Observed measures are shown in boxes for PBI and CESD measures on a pair of twins ( $T 1$ and $T 2$ ). These variables are caused by additive genetic (A), common environment (C), and random environment (E) factors. Also, latent factors for CESD may cause variation in PBI (paths $a_{p c}, c_{p c}$, and $e_{p c}$ ), and PBI and CESD may have direct causal influence on each other (paths $i_{c}$ and $i_{p}$ ). Only 3 of these across-variable parameters may be estimated simultaneously. $\alpha$ is fixed at 1.0 for $\mathrm{MZ}$ twins and 0.5 for $\mathrm{DZ}$ twins.

though the bidirectional and common $\mathrm{C}$ models are often almost as parsimonious. The models with an asterisk $(*)$ are unidirectional causal models that incorporate an additional parameter to estimate error of measurement for the causal variable. While these models offer a more parsimonious explanation of the data than models without measurement error, they still do not account for the data as efficiently as the model with common additive genetic effects alone.

For certain variables an improved fit of the bidirectional model could be obtained if parameters were allowed to take values that would lead to unstable feedback loops. This will arise if the matrix of reciprocal paths between variables (B) has eigenvalues that lie outside the unit circle (i.e., the sum of the squares of the real and imaginary components is greater than one). To steer estimation away from these conditions, $\mathrm{Mx}$ constraint functions were specified to constrain the eigenvalues of $\mathrm{BB}^{\prime}$ to be less than unity. No error of measurement was specified for either variable in this model.

Parameter estimates of the common A model and the unidirectional causation with error models (Table IV) show the slightly lower resemblance between rearing style and depression than found in other studies. Although common genetic variance $\left(a_{R D}^{2}\right)$ is quite low, the model predicts a correlation of $a_{P C} a_{C}$ between the variables, or 0.23 for paternal coldness. The values of the paths in the causal models with error exceed this value, but again predict a similar between-variable within-person correlation. For example, with the CESD* $\rightarrow$ FACOLD model the within-person correlation is calculated as $\left(a_{C}^{2}+c_{C}^{2}+e_{C}^{2}\right) i_{C}=0.24$. The estimates of error are greater for CESD than for ratings, a paradoxical result because CESD has many more items. However, as the proportion of error variance in ratings increases, so does the 


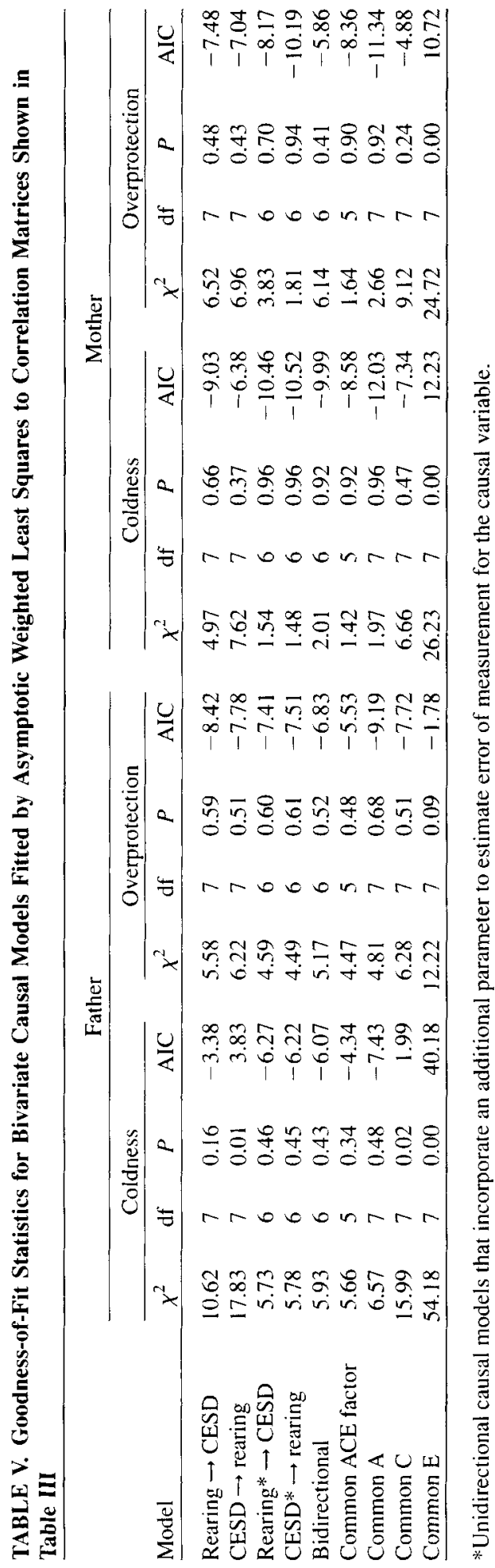




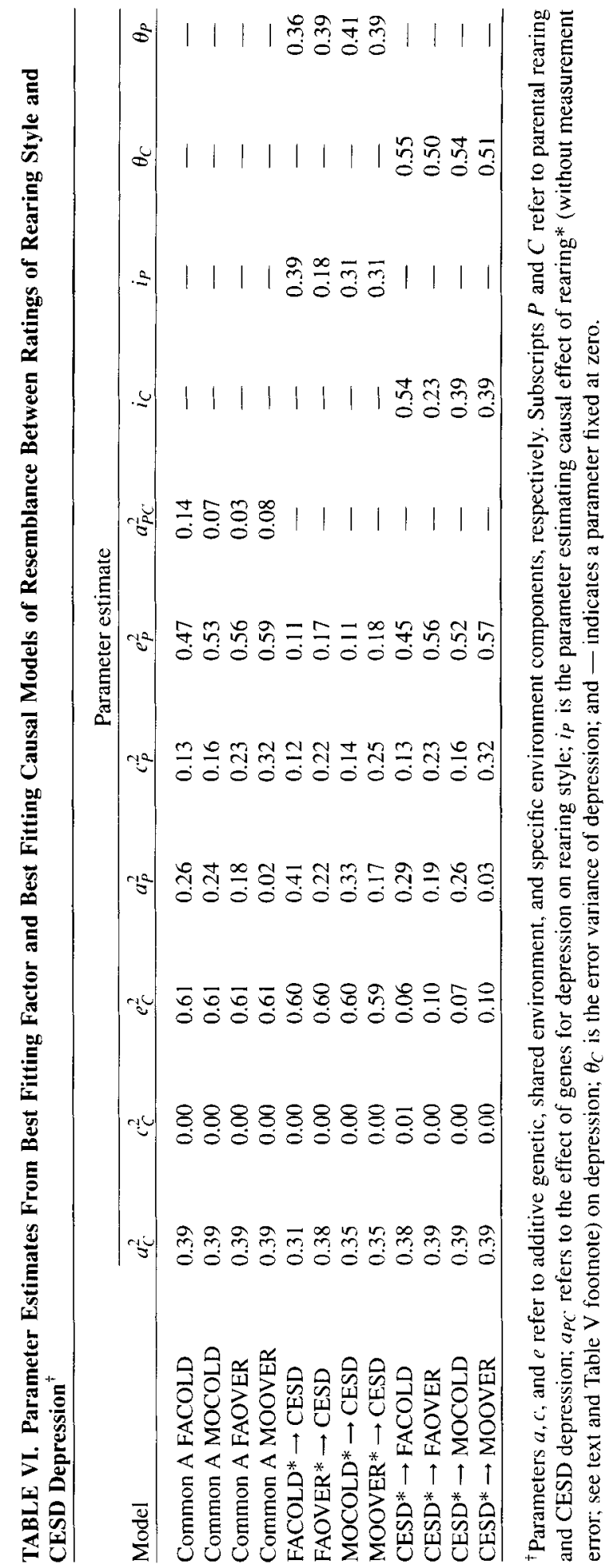


proportion of shared environmental variance in the transmitted or causal component, which in turn would predict shared environmental variance in CESD. The univariate analysis strongly suggests that this is absent, so the estimates favor less error for ratings than for CESD. The estimates of error are broadly equivalent across all 4 measures of parental rearing style, suggesting that the limited number of items for overprotection does not seriously compromise its measurement.

\section{Model for Eliciting Parental Rearing}

Imagine that children elicit particular rearing styles from their parents. Such behavior by children, being a personality trait, would be likely to have some genetic variance [Eaves et al., 1978, 1989; Heath et al., 1989; Jinks and Fulker, 1970; Neale et al., 1986; Young et al., 1980]. If this were the case, then MZ twins would be more similar than $\mathrm{DZ}$ twins, and we would predict increased variance of parental treatment in the parents of $\mathrm{MZ}$ twins relative to the parents of DZ twins (see Fig. 5). To test for such heterogeneity, contingency tables of the responses of twin 1 and twin 2 were constructed separately for $\mathrm{MZ}$ and $\mathrm{DZ}$ groups. This was done for each of the 7 items that twins were asked to answer about both parents (data available on request). In a accordance with the central limit theorem, we assume that there is a normal distribution of liability of response, with abrupt thresholds separating the response classes. This liability is allowed to correlate between twins. In effect, group differences in the variance of the underlying scale translate into differences in the thresholds, such that if $q$ is the ratio of the standard deviation of $\mathrm{MZ}$ twins to the standard deviation of DZ twins, the standardized thresholds of $\mathrm{DZ}$ twins $\left(t_{\mathrm{DZ} i}\right)$ are predicted to be $t_{\mathrm{MZi}} / q$. The difference in log-likelihood between a model in which the variances of $\mathrm{MZ}$ and $\mathrm{DZ}$ ratings differ and one in which the variances are constrained to be equal provides the required test of heterogeneity. These tests were performed

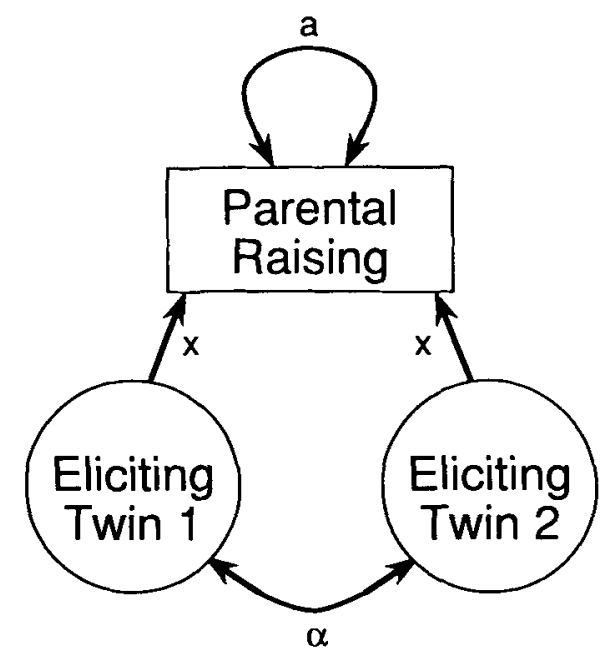

Fig. 5. Model for eliciting rearing responses from parents. The path $x$ represents the regression of rearing on the latent variable for eliciting by the child. The parameters $\alpha$ may vary according to the zygosity of the twins if eliciting behavior is partly genetic in origin. The predicted variance of rearing is $a+2 x^{2}+2 x^{2} \alpha$, and differs between parents of MZ and DZ twins if eliciting is partly genetic and $\alpha$ is non-zero. 
separately for all 7 items using Mx [Neale, 1991] and the results are shown in Table VII. We recognize that these tests are not independent, but the equivalent multivariate test would require integration of a 28-dimensional multivariate normal distribution which is beyond practical limitations of current software and hardware for numerical quadrature. There appears to be little evidence for children eliciting parental behavior in these data. Some caution is required because the power of this test is not great, especially with categorical data which are imprecisely known.

\section{DISCUSSION}

Causal hypotheses lead to predictions for covariation between variables both across time and across relatives. The choice of design for testing these hypotheses will often depend on characteristics of the variables under study, since each design has its own advantages and disadvantages. For example, if we know that 2 variables develop a strong covariance during a particular period of development, then a longitudinal study spanning this ontogeny is appropriate. Conversely, if 1 variable has delayed onset or if observation at 1 or more time points is impractical, a cross-sectional study of pairs of relatives may be more suitable. One disadvantage of the longitudinal design is the ethical one, since subjects (especially children) discovered to be depressed on the first occasion of method should be referred for treatment. This referral would reduce the similarity between the sample studied and the population in its natural state. If directions of causation between the variables of interest and a third variable are known, this additional variable may be used as an instrumental variable to test causal models. However, this third approach is not often appropriate in the social sciences due to the complexity of its subject matter and the relative paucity of high quality data.

The possible presence of mediating variables that could mimic the statistical effects of causation has led to criticism of longitudinal methods [Kenny, 1975; Rogosa, 1980; see also Locasio, 1982]. The models discussed here for cross-sectional data make similar assumptions about the independence of the sources of variation in each variable, so they share this same weakness. However, with data from several different classes of relative, some of this deficiency may be ameliorated by using combined models that allow for both causal influence and some shared genetic or environmental

TABLE VII. Tests for Heterogeneity of Item Variance Between Ratings of Parents From MZ $(N=490$ Pairs $)$ and DZ $(N=336$ Pairs $)$ Twins*

\begin{tabular}{lcc}
\hline Item & Fathers & Mothers \\
\hline Let me do things I liked & $\mathbf{0 . 3 6}$ & 0.96 \\
Emotionally cold to me & 0.26 & 3.53 \\
Understood my problems & $\mathbf{0 . 1 0}$ & 0.59 \\
Liked me to make own decisions & 1.75 & 3.64 \\
Made me feel not wanted & 0.02 & 4.18 \\
Made me dependent on her & $\mathbf{1 . 4 7}$ & $\mathbf{0 . 2 4}$ \\
Was overprotective & 0.02 & $\mathbf{0 . 6 5}$ \\
\hline
\end{tabular}

*All statistics are $\chi^{2}$ difference, with one degree of freedom. Boldface numbers indicate that the variance of ratings by MZs is estimated to be greater than the variance of ratings by DZs, though none of these achieves statistical significance. 
factors. It can be shown that any 3 of the 5 postulated parameters for covariation between variables (shared genetic, common, or specific environment effects, and bidirectional causation) may be estimated with data from $\mathrm{MZ}$ and $\mathrm{DZ}$ twins, but the power to resolve parameters in these mixed models is likely to be very low indeed for most real variables. This is also the case when models for measurement error are incorporated into the directional causal models, though the use of multiple indicators (either similar instruments or retest data) may often overcome this disadvantage. In the long run, the optimum approach may be to combine both longitudinal and genetically informative methods in a longitudinal behavior genetic design [Eaves et al., 1986; McArdle, 1986]. Our confidence in rejecting or supporting hypotheses is greatly increased if these 2 very different approaches lead to the same conclusions.

Application of direction of causation models to data from twins on the PBI and CESD rejects the hypothesis that depression causes ratings of paternal coldness. This suggests that ratings are not simply due to differences in recall bias associated with liability to depression. For maternal coldness and overprotectiveness in both parents, statistical rejection of the depression-causes-ratings model was not achieved, but in each case the reversed model (ratings-cause-depression) is more strongly supported by the data. Furthermore, statistical rejection of the causal models was not possible once error of measurement was incorporated in the model. The most parsimonious explanation of the data is given by a simple model that postulates all covariance to be due to genetic factors that affect both depression and ratings of parental rearing. We can make at least 3 interpretations of this result. First, there are genetic factors that contribute both to rearing style and to depression. These genetic factors are transmitted from parent to child and result in the association between depression and (reported) rearing style in the parent. A second possibility is that there are genetic factors in depression that also cause variation in rating style. Although there is a moderate correlation between parents' self-report of rearing style and reports by their children [Parker, 1981], there is still room for considerable error by either rater. Recently we have collected data on the PBI and depression from the parents of the twins in this study. These data will help to discriminate between the effects of rater bias by examining the covariance between parents' self-ratings and ratings by their twin children [Neale and Stevenson, 1988]. In addition, using multivariate analysis of familial resemblance we should be able to discriminate between parent to child cultural transmission, pleiotropic genetic factors, and effects of assortative mating [Neale and Fulker, 1984; Vogler, 1985; Carey, 1986]. A third interpretation is that genetic factors are the most significant in the relationship between ratings of parents and depression, but there is also some causal impact from parent to child, and some rating bias by children, although the sample size used here is not sufficient to establish the presence of these different factors. The difference in fit between the unidirectional with error model and the common A model is quite small, especially for the coldness scales. Neither model fits the data significantly worse than the common ACE model, and the confidence intervals on the $\chi^{2}$ statistics are large. The precision of the asymptotic weight matrix computer by PRELIS 1.20 is also open to question since this software is under revision [Rigdon and Ferguson, 1991]. At the extreme end, coldness and overprotectiveness would constitute child abuse. The effects of such extreme forms of parental treatment may not be reflected in these data 
from a population-based sample and could have more direct and dire consequences for development than the data presented here suggest.

Twin resemblance for the CESD scale indicates a modest heritability (26\%) with a non-significant proportion of common environmental variance $(11 \%)$, and the most parsimonious model estimates heritability at $39 \%$ with no effect of the common environment. These results are quite consistent with most other published studies of depression [Kendler et al., 1991b]. The correlation between ratings of rearing and CESD are somewhat lower than most studies; this could be a consequence of using a short form of the scale, though preliminary results from a telephone follow-up of this sample would suggest otherwise. The CESD scales correlate $0.11,0.18,0.20$, and 0.16 with maternal $(N=1,463)$ and paternal $(N=1,443)$ coldness and maternal and paternal overprotectiveness, respectively. Here at least part of the low value would seem to be due to change in ratings (or unreliability) over time. The test-retest correlations for the short scales are 0.66 for maternal and paternal coldness $(N=1,580)$ and 0.62 for maternal and 0.55 for paternal overprotectiveness.

The correlations between twins for ratings of coldness and overprotectiveness in mothers and fathers are lower than those found using the full scale in a small sample [Parker, 1986]. This could be due to the reduced number of items (particularly for overprotectiveness) or differences between populations or sampling error in either sample. The current study reveals some evidence for shared environmental affects in ratings of mothers, which is to be expected since twins share the same mother! However, despite sharing the same father, only genetic and random environmental variance appears significant for paternal rearing styles. Such a pattern would be observed if fathers were treating their children differentially [Daniels and Plomin, 1985]--possibly in response to genetic differences in the children. This scenario might be predicted from sociobiological principles, since fathers have less confidence about their biological relatedness to their children than do mothers [Alexander, 1979]. If indeed parents respond differentially to their children's phenotypes, and variation in these phenotypes is partly genetic, then differences in total variance of parents' rearing styles are predicted according to zygosity. However, no such differences were found, possibly because of the relatively weak power of the design, which is exacerbated by the use of categorical data.

Finally, there is the possibility of genotype by environment $(G \times E)$ interaction. It is plausible that there are genetic predispositions to depression that are only expressed under certain environmental conditions. Different environments have been shown to elicit expression of different genes in plant and non-human animal species [Mather and Jinks, 1982] and similar processes have been demonstrated for certain human phenotypes [Heath, 1986; Heath et al., 1989; Kendler et al., 1991b]. Genetic analysis of measures of depression contingent on exposure to putative environmental factors such as the presence or absence of adverse rearing experiences is an important area for future research. However, since ratings of rearing style are neither purely environmental nor genetically independent of measures of depression, analysis of the interaction between these phenotypes will not be a simple matter.

\section{ACKNOWLEDGMENTS}

Data collection was supported by ADAMHA grant $\mathrm{MH}-40828$. Data analysis was also supported by grants AG-04954 and MH-45268. The Virginia Twin Registry 
was established and is maintained by Drs. W.E. Nance and L. Corey, and is supported by NIH grants HD-26746 and NS-25630.

\section{REFERENCES}

Akaike H (1987): Factor analysis and AIC. Psychometrika 52:317-332.

Alexander RD (1979): "Darwinism and Human Affairs." Seattle: University of Washington Press.

Alnaes R, Torgerson S (1990): Parental representations in patients with major depression, anxiety disorder, and mixed conditions. Acta Psychiatr Scand 81:518-522.

Beck AT, Rush AJ, Shaw BF, Emery G (1979): "Cognitive Theory of Depression." New York: Gilford Press.

Becker J (1974): "Depression-Theory and Research.” Winston, WA

Biddle BJ, Slavings RL, Anderson DS (1985): Methodological observations on apflied behavioral science. J Appl Behav Sci 21:79-93.

Birtchnell J (1988): Depression and family relationships: A study of young married women on a London housing estate. Br J Psychiatry 153:758-769.

Bollen KA (1989): "Structural Equations With Latent Variables." New York: John Wiley \& Sons.

Browne MW (1984): Asymptotically distribution-free methods for the analysis of covariance structures. Br J Math Stat Psychol 37:1-21.

Campbell DT (1963): From description to experimentation: Interpreting trends from quasi-experiments. In Harris CW (ed): "Problems in Measuring Change." Madison: University of Wisconsin Press.

Carey G (1986): A general multivariate approach to linear modelling in human genetics. Am J Hum Genet 39:775-786.

Chodoff P (1972): The depressive personality. Arch Gen Psychiatry 27:666-673.

Comstock GW, Helsing KJ (1976): Symptoms of depression in two communities. Psychol Med 6:551-563.

Costello CG, Comrey AL (1967): Scales for measuring depression and anxiety. J Psychol 66:303--313.

Daniels D, Plomin R (1985): Differential experience of siblings reared in the same family. Dev Psychol $21: 747-760$

Duffy DA, Martin NG (1994): Inferring the direction of causation in cross-sectional twin data: Theoretical and empirical considerations. Genet Epidemiol 11:483-502.

Eaves LJ, Long J, Heath AC (1986): A theory of developmental change in quantitative phenotypes applied to cognitive development. Behav Genet 16:143-162.

Eaves LJ, Last K, Young PA, Martin NG (1978): Model fitting approaches to the analysis of human behaviour. Heredity 41:249-320.

Eaves LJ, Eysenck HJ, Martin NG (1989): "Genes, Culture and Personality: An Empirical Approach." London: Academic Press.

Gotlib IH, Mount JH, Cordy NI, Whiffen VE (1988): Depression and perceptions of early parenting: A longitudinal investigation. Br J Psychiatry 152:24-27.

Heath AC (1986): Detecting the effects of genotypes $\times$ environment interaction on personality and symptoms of depression. Behav Genet 16:622.

Heath AC, Neale MC, Hewitt JK, Eaves LJ, Fulker DW (1989): Testing structural equation models for twin data using LISREL. Behav Genet 19:9-36.

Heise DR (1975): "Causal Analysis.” New York: John Wiley \& Sons.

Holmes SJ, Robins LN (1987): The influence of childhood disciplinary experience on the development of alcoholism and depression. J Child Psychol Psychiatry 28:399-415.

Jacobson S, Fasman J, DiMascio A (1975): Deprivation in the childhood of depressed women. J Nerv Ment Dis 160:5-14.

Jinks JL, Fulker DW (1970): Comparison of the biometrical genetical, MAVA and classical approaches to the analysis of human behavior. Psychol Bull 73:311-349.

Jöreskog KG, Sörbom D (1986): "PRELIS: A Preprocessor for LISREL." Chicago: Scientific Software, Inc.

Kendler KS, Kidd KK (1986): Recurrence risks in an oligogenic threshold model: The effect of alterations in allele frequency. Ann Hum Genet 50:83-91.

Kendler KS, Kessler RC, Heath AC, Neale MC, Eaves LJ (1991a): Coping: A genetic epidemiologic investigation. Psychol Med (in press). 
Kendler KS, Neale MC, Heath AC, Kessler RC, Eaves LJ (199lb): Life events and depressive symptoms: A twin study perspective. In McGuffin P, Murray RM (eds): "The New Genetics of Mental Illness." London: Butterworth Heinemann.

Kenny DA (1975): Cross-lagged panel correlations: A test for spuriousness. Psychol Bull 82:887-903.

Kessler RC, Greenberg DF (1981): "Linear Panel Analysis: Models of Quantitative Change." New York: Academic Press.

Lewinsohn PM, Rosenbaum M (1987): Recall of parental behavior by acute depressives, remitted depressives, and non-depressives. J Person Soc Psychol 52:611-619.

Li CC (1975): "Path Analysis-A Primer." Pacific Grove. Boxwood Press.

Locasio JJ (1982): The cross-lagged correlation technique: Reconsideration in terms of an exploratory utility, assumption specification and robustness. Educ Psychol Meas 42:1023-1036.

Mackinnon AJ, Henderson AS, Scott R, Duncan-Jones P (1989): The Parental Bonding Instrument (PBI): An epidemiological study in a general population sample. Psychol Med 19:1023-1034.

Mardia KV, Kent JT, Bibby JM (1979): “Multivariate Analysis.” New York: Academic Press.

Martin NG, Eaves LJ (1977): The genetical analysis of covariance structures. Heredity 38:79-95.

Mather K, Jinks JL (1982): "Biometrical Genetics.” 3rd Ed. New York: Methuen.

Mayer LS (1984): "Statistical Inferences in Cross-lagged Panel Studies." Technical report. Stanford, CA: Department of Statistics, Stanford University.

McArdle JJ (1986): Latent variable growth within behavior genetic models. Behav Genet 16:163-200.

McArdle JJ, Boker SM (1990): "RAMpath: Path Diagram Software." Denver: Data Transforms, Inc.

Morris-Yates A, Andrews G, Howie P, Henderson S (1990): Twins: A test of the equal environments assumption. Acta Psychiat Scand 81:322-326.

Myers JK, Weissman MM (1980): Use of self-report symptom scale to detect depression in a community sample. Am J Psychiatry 137:1081-1084.

Neale MC (1988): Handedness in a sample of volunteer twins. Behav Genet 18:69-79.

Neale MC (1991): "Mx: Statistical Modeling: User's Guide." Richmond: Department of Human Genetics.

Neale MC, Cardon LR (1992): "Methodology for Genetics Studies of Twins and Families." The Netherlands: Kluwer Academic Publishers.

Neale MC, Fulker DW (1984): A bivariate path analysis of fear data on twins and their parents. Acta Genet Med Gemellol 33:237-286.

Neale MC, Martin NG (1989): The effects of age, sex and genotype on self-report drunkenness following a challenge dose of alcohol. Behav Genet 19:63-78.

Neale MC, Stevenson J (1989); Rater bias in the EASI temperament scales: A twin study. J Person Soc Psychol 56:446-455.

Neale MC, Rushton JP, Fulker DW (1986): The heritability of items from the Eysenck Personality Questionnaire. Person Indiv Diff 7:771-779.

Neale MC, Heath AC, Hewitt JK, Eaves LJ, Fulker DW (1989): Fitting genetic models with LISREL: Hypothesis testing. Behav Genet 19:37-49.

Parker G (1979a): Reported parental characteristics in relation to trait depression and anxiety levels in a non-clinical group. Aust NZ J Psychiatry 13:260-264.

Parker G (1979b): Parental characteristics in relation to depressive disorders. Br J Psychiatry 134:138-147.

Parker G (1981): Parental reports of depressives: An investigation of several explanations. J Affect Disord 3:131-140.

Parker G (1986): Validating an experimental measure of parental style: The use of a twin sample. Acta Psychiatr Scand 73:22-27.

Parker G, Kiloh L, Hayward L (1987): Parental representations of neurotic and endogenous depressives. J Affect Disord 13:75-82.

Perris C, Maj M, Perris H, Eisemann M (1985): Perceived parental rearing behaviour in unipolar and bipolar depressed patients. Acta Psychiat Scand 72:172-175.

Plantes MM, Prusoff BA, Brennan J, Parker G (1988): Parental representations of depressive outpatients from a USA sample. J Affect Disord 15:149-155.

Plomin R, McCleam GE, Pedersen NL, Nesselroade JR, Bergeman CS (1988): Genetic influence on childhood family environment perceived retrospectively from the last half of the life span. Dev Psychol 24:738-745.

Radloff LS (1977): The CES-scale: A self-report depression scale for research in the general population. Appl Psychol Meas 3:385-401. 
Raskin A, Boothe HH, Reatig NA, Schulterbrandt JG, Odle D (1971): Factor analyses of normal and depressed patients' memories of parental behavior. Psychol Rep 29:871-879.

Rigdon EE, Ferguson CE (1991): The performance of the polychoric correlation coefficient and selected fitting functions in confirmatory factor analysis with ordinal variables. J Market Res 28:491-497.

Robins LN, Schoenberg SP, Holmes SJ, Ratcliff KS, Benham A, Works J (1985): Early home environment and retrospective recall: A test for concordance between siblings with an without psychiatric disorders. Am J Orthopsychiatry 55:27-41.

Rogosa DA (1980): A critique of cross-lagged correlation. Psychol Bull 88:245-258.

Rutter M (1986): The developmental psychopathology of depression: Issues and perspectives. In Rutter M, Izard CE, Read PB (eds): "Depression in Young People." New York: Guilford Press.

Theil H (197I): "Principles of Econometrics." New York: John Wiley \& Sons.

Vogler GP (1985): Multivariate path analysis of familial resemblance. Genet Epidemiol 2:35-54.

Werts CE, Linn RL, Jöreskog KG (1971): Estimating the parameters of path models involving unmeasured variables. In Blalock HM (ed): "Causal Models in the Social Sciences." Chicago: Aldine-Atherton.

Werts CE, Linn RL, Jöreskog KG (1977): A simplex model for analyzing academic growth. Educ Psychol Meas 38:89-95.

Werts CE, Jöreskog KG, Linn RL (1985): Comment on the estimation of measurement error in panel data. In Blalock HM Jr (ed): "Causal Models in Panel and Experimental Designs." New York: Aldine, pp $145-150$.

Wilhelm K, Parker G (1990): Reliability of the parental bonding instrument and intimate bond measure scales. Aust NZ Psychiatry 24:199-202.

Young PA, Eaves LJ, Eysenck HJ (1980): Intergenerational stability and change in the causes of variation in personality. Person Indiv Diff 1:35-55.

\section{APPENDIX}

The following procedure for imputing items was used for imputing item responses on scales for which most items had been answered.

\section{Imputation Scheme}

Scores will be imputed for individuals who answer at least a threshold number of items. The threshold is defined as the next highest integer above half the total number of items $(N)$ in the scale. For all individuals who completed all items, the total score is converted to a $(0,1)$ scale by the following operation: (score - minimum possible score)/(maximum possible score - minimum possible score). Based on these individuals, a mean and a standard deviation are computed for each item in the scale $\left(\bar{x}_{i} \pm s d_{i}\right)$ where $i=i \ldots N$. The mean and standard deviation of the total scale score are also computed $\left(\bar{x}_{T} \pm s d_{T}\right)$. For each individual who did not answer all items but answered at least the threshold, a standardized deviation from the mean is computed for each item answered $\left(\left(x_{i}-\bar{x}_{i}\right) / s d_{i}\right)$. For each individual, themean of these standardized deviations is then computed $\left(x^{\prime}\right)$ and the imputed score is calculated as $\bar{x}_{T}+\left(x^{\prime}\right)\left(s d_{T}\right)$. If the imputed score is less than zero, the score is set to zero and if the imputed score is greater than one, the score is set to one.

Data are complete for most subjects in this sample. CESD scores were imputed for 188 of 2,139 individuals, of whom 149 were missing only 1 item and 26 were missing 2 items. Twenty-nine subjects were missing 1 item from the maternal coldness scale, 30 were missing 1 item from the paternal scale, and 2 were missing 2 items. 